\title{
Influence of the interaction potential on simulated sputtering and reflection data
}

\author{
W. Eckstein ${ }^{1}$, S. Hackel ${ }^{2}$, D. Heinemann ${ }^{2}$, B. Fricke ${ }^{2}$ \\ ${ }^{1}$ Max-Planck-Institut für Plasmaphysik, EURATOM-Association, W-8046 Garching, Federal Republic of Germany \\ ${ }^{2}$ Universität Kassel, W-3500 Kassel, Federal Republic of Germany
}

Received: 18 October 1991 / Final version: 23 March 1992

\begin{abstract}
The TRIM.SP program which is based on the binary collision approximation was changed to handle not only repulsive interaction potentials, but also potentials with an attractive part. Sputtering yields, average depth and reflection coefficients calculated with four different potentials are compared. Three purely repulsive potentials (Molière, $\mathrm{Kr}-\mathrm{C}$ and $\mathrm{ZBL}$ ) are used and an ab initio pair potential, which is especially calculated for silicon bombardment by silicon. The general trends in the calculated results are similar for all potentials applied, but differences between the repulsive potentials and the ab initio potential occur for the reflection coefficients and the sputtering yield at large angles of incidence.
\end{abstract}

PACS: 79.20.; 34.20.

\section{Introduction}

In simulation programs based on the binary collision approximation only repulsive interaction potentials have been used so far, despite the fact that atoms in the groundstate usually attract each other above some distance. The argument for purely repulsive potentials is the low potential energy at larger distances. This is certainly justified at large particle energies, but at low energies, which have to be considered for sputtering calculations, larger internuclear separations become important. The attractive potential well is of the order of the surface binding energy, which indicates a possible influence on the sputtering yield and on the reflection coefficients especially at grazing incidence. The attractive potential also leads to negative scattering angles at large interatomic distances. The question is if this different scattering behaviour will cancel out during the many collisions which usually occur during the development of the cascade. At low energies this influence of an attractive part in the potential is expected to be larger than at higher energies because of the limited number of collisions and the lower potential energy.

This paper discusses a comparison of calculated range, sputtering and reflection data using three repulsive potentials and a potential especially determined by the Hartree-Fock-Slater method for the bombardment of silicon by silicon.

The main emphasis is put on possible differences in the results achieved with the repulsive potentials and a potential with an attractive part. It is not the intention to prove that a specific potential gives the best agreement with experimental data. More complicated potentials like the Stillinger-Weber [1] and Tersoff [2] potentials have been developed where even threebody interactions (at least implicity) have been included to describe the silicon solid satisfactorily. For the case of sputtering Harrison [3] has studied the dependence of the yield on different potentials. A more complete survey of interaction potentials is given in [4].

\section{Interaction potentials}

The interaction potentials chosen are three repulsive potentials: The Molière- [5], the krypton-carbon ( $\mathrm{KrC}$ )[6] and the Ziegler-Biersack-Littmark ZBL (sometimes called the 'universal') [7] potential. In the Molière- and KrC-potentials the Firsov screening length, $a_{F}$, is used

$a_{F}=0.8853 a_{B}\left(Z_{1}^{1 / 2}+Z_{2}^{1 / 2}\right)^{-2 / 3}$,

whereas the ZBL screening length is given by

$a_{Z \mathrm{BL}}=0.8853 a_{B}\left(Z_{1}^{0.23}+Z_{2}^{0.23}\right)^{-1}$,

where $a_{B}$ is the Bohr radius.

All three potentials have the general form

$V(r)=\frac{Z_{1} Z_{2} e^{2}}{r} \Phi\left(\frac{r}{a}\right)$ 
Table 1. Constants in the screening functions for various potentials (4)

\begin{tabular}{llll}
\hline & $\begin{array}{l}\text { Molière } \\
{[5]}\end{array}$ & $\begin{array}{l}\mathrm{Kr}-\mathrm{C} \\
{[6]}\end{array}$ & $\begin{array}{l}\mathrm{ZBL} \\
{[7]}\end{array}$ \\
$n$ & 3 & 3 & 4 \\
\hline$c_{1}$ & 0.35 & 0.190945 & 0.02817 \\
$c_{2}$ & 0.55 & 0.473674 & 0.28022 \\
$c_{3}$ & 0.10 & 0.335381 & 0.50986 \\
$c_{4}$ & 0 & 0 & 0.18175 \\
$d_{1}$ & 0.3 & 0.278544 & 0.20162 \\
$d_{2}$ & 1.2 & 0.637174 & 0.40290 \\
$d_{3}$ & 6.0 & 1.919249 & 0.94229 \\
$d_{4}$ & 0 & 0 & 3.1998 \\
\hline
\end{tabular}

with the screening function

$\Phi\left(\frac{r}{a}\right)=\sum_{i=1}^{n} c_{i} \exp \left(-d_{i} \frac{r}{a}\right), \quad \sum_{i=1}^{n} c_{i}=1$.

The constants $c_{i}$ and $d_{i}$ are given in Table $\mathrm{l}$.

The Molière potential is an empirical fit to the ThomasFermi function; the $\mathrm{KrC}$ potential describes the interaction between $\mathrm{Kr}$ and $\mathrm{C}$ atoms, but has shown to be a good mean potential for many different atom-atom-collisions [8]. The ZBL potential is determined as a mean potential of many individual cases calculated by a Hartree-Fock-Slater method (non relativistic).

An ab initio calculation using a finite-element molecular self-consistent field Hartree-Fock-Slater (SCF-HFS) code [9] gives the interaction between two silicon atoms [10]. This pointwise determined interaction potential is approximated by

$$
\begin{aligned}
\Phi\left(r / a_{F}\right)= & \left\{0.35 \exp \left(-0.28 r / a_{F}\right)\right. \\
& +0.55 \exp \left(-1.2 r / a_{F}\right)+0.1 \exp \left(-6 r / a_{r}\right) \\
& \left.-0.002327\left(r / a_{F}\right) \exp \left[-0.15\left(r / a_{r}-5.757\right)^{2}\right]\right\} \\
& \times\left[1-0.005713\left(r / a_{F}\right)^{2}\right]
\end{aligned}
$$

The approximation is based on the Molière potential with additional terms to account for the zero crossing and the attractive potential well. The well depth is slightly smaller in the approximation than in the pointwise determined potential.

A comparison of the potential functions used is shown in Fig. 1. At small distances all potentials coincide, then with increasing internuclear separation the Si-Si potential follows the $\mathrm{KrC}$, then switching to the $\mathrm{ZBL}$ potential, reaching zero at about $1.6 \AA$ and the minimum at about $2 \AA$.

It should be mentioned that the Si-Si potential calculated here is based on atoms in the ground state. Any excitation or ionization would change the potential function especially at larger internuclear separations of the two atoms. It may very well be that the potential which actually occurs in a realistic case is somewhere between the ab initio groundstate potential and the empirical potentials. Nevertheless, it is important to demonstrate the
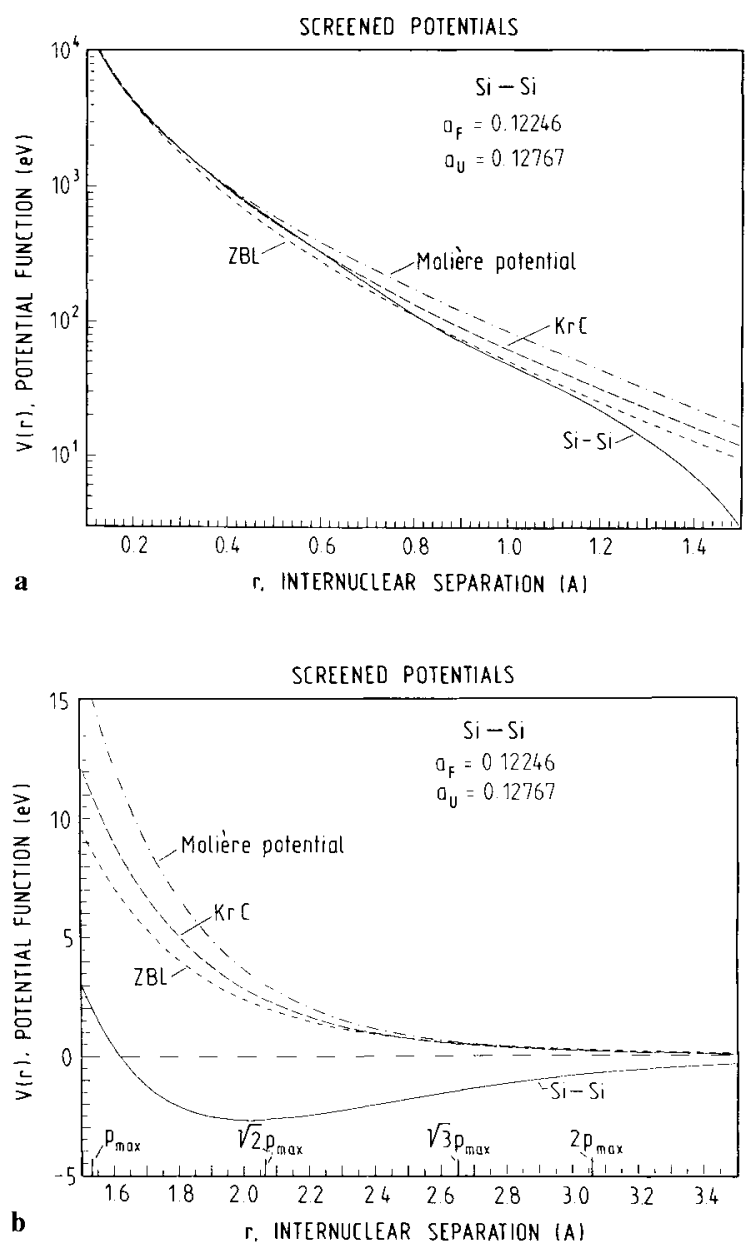

Fig. 1a, b. Four different silicon-silicon interaction potentials: Molière, Krypton-carbon ( $\mathrm{KrC}$ ), Ziegler-Biersack-Littmark ( $\mathrm{ZBL})$ and a specific silicon-silicon ( $\mathrm{Si}-\mathrm{Si}$ ) potential, see (5). The Firsov screening length $a_{F}$ is used in Molière, $\mathrm{KrC}$ and Si-Si potentials, $a_{\mathrm{ZBL}}$ in the ZBL potential. a small internuclear separations. b large internuclear separations; the ring distances $\sqrt{n} P_{\max }$ (see text) are indicated

dependence of the physical observables from the various choices of the interaction potential. From that point of view the ab initio calculated potential must not necessarily be better than an empirical potential.

\section{The calculational model}

The influence of different interaction potentials on range, backscattering and sputtering data is determined by the Monte Carlo program TRIM.SP (version TESTSI). The program TRIM.SP is described in detail in [11]. The main essentials are: the solid has a randomized structure, it is based on the binary collision approximation, the elastic collisions are described by one of the interaction potentials mentioned above, energy losses due to electrons are treated as an equipartition of the local Oen-Robinson model [12] and the nonlocal Lindhard-Scharff model [13], a planar surface binding potential $(4.70 \mathrm{eV})$ leads to at refraction and an energy loss (gain) of emitted (incident) particles. The cutoff energy is taken slightly below the surface binding energy, namely $4.6 \mathrm{eV}$. 


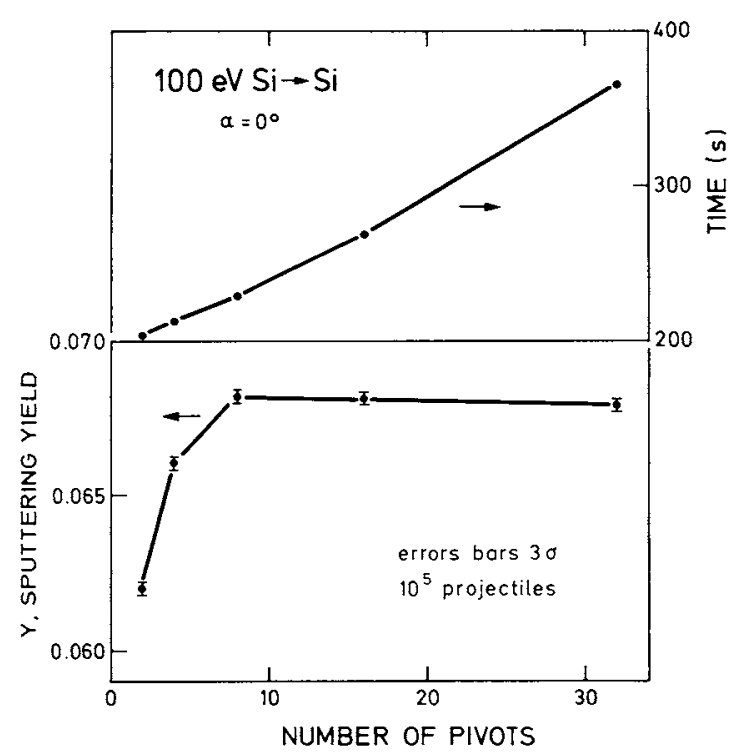

Fig. 2. Computing time and sputtering yield, $Y$, versus the number of pivots for the integration procedure. Silicon is bombarded by $100 \mathrm{eV} \mathrm{Si}$ at normal incidence, the $\mathrm{KrC}$ potential is used

In contrast to the original program [11] the new version is vectorized which means a complete restructuring. In the new version the density is conserved. The integration procedure 'Magic' [14] applied in TRIM.SP works only for repulsive potentials. Therefore in version TESTSI the scattering angle and the time integral are determined by the Gauss-Mehler integration procedure [15] which is also used in the MARLOWE program [16] and for the calculation of scattering data in tables $[17,18]$. The accuracy of the calculated scattering angle and the time integral depends on the number of pivots and is discussed in [18]. In Fig. 2 the dependence of the calculation time and the sputtering yield, $Y$, on the number of pivots used is shown for the example of $10^{5}$ silicon atoms with an energy of $100 \mathrm{eV}$ at normal incidence $\left(\alpha=0^{\circ}\right)$ on a silicon target. For the number of pivots larger than 8 , the sputtering yield does not change in the statistical limits. In all calculations given below, 16 pivots have been used which gives an increase in computing time of about $30 \%$ compared to 4 pivots. The increase in the sputtering yield of about $10 \%$ from 2 pivots to 8 pivots is usually in the error limits of experimental data. In MARLOWE calculations often 4 pivots are used.

As described in $[4,11]$, simultaneous weak collisions are applied in TRIM.SP to account for the fact that at low energies a particle may interact not only with one atom (as in the binary collision approximation). A collision partner is searched for in a cylindrical volume with $P_{\max }$ as the radius. Also additional collision partners (weak collisions) are randomly chosen in concentric successive ring cylinders with the same volume. The elastic energy losses of these simultaneous collisions and the corresponding scattering angles are taken into account as corrections to the values found for the cylindrical volume. In other words, the resulting scattering angle and energy loss of a moving $\mathrm{Si}$ atom with the solid is a superposition of all collision events with the partners from
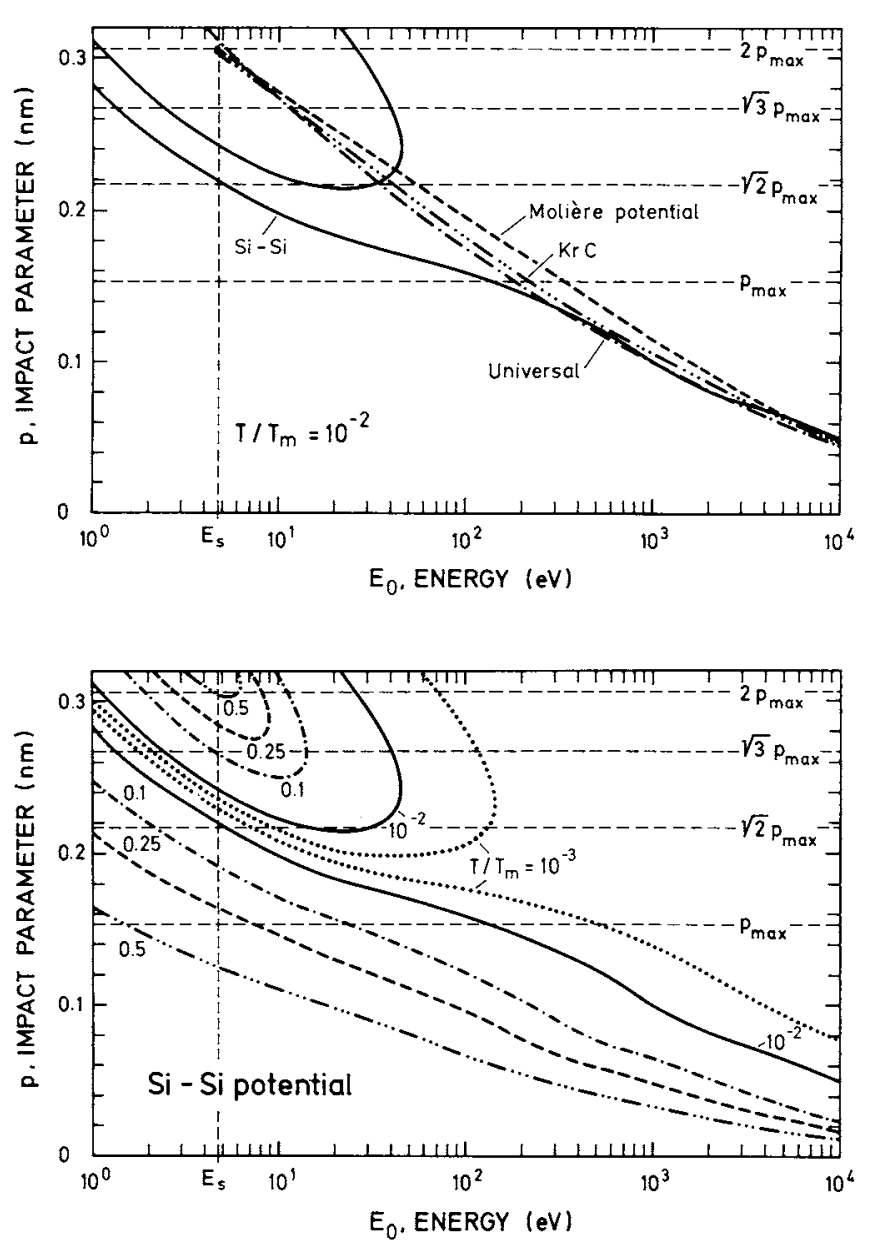

Fig. 3a, b. Impact parameter, $p$, versus the energy, $E_{0} . T / T_{i: 1}$ is the relative energy loss ( $T_{m}=1$ for equal collision partners) for $\mathrm{Si}-\mathrm{Si}$ collisions. The values for the maximum impact parameters in the search cylinder $\left(p_{\max }\right)$ and the successive ring cylinders are indicated. a $T / T_{m}=10^{-2}$ is fixed. Curves for different potentials are given. b Curves for several values of $T / T_{m}$ for the Si-Si potential

the inner cylinder and the ring cylinders. In doing so we use the approximation that the effective potential of the atom-solid interaction can be broken up in a sum of atomatom potentials. Thus we neglect the higher order effect of the rearrangement of the potential in the many atom case. In detail we proceed as follows. As shown before [11], for repulsive potentials it is sufficient to take two ring cylinders into account. A further weak collision does not influence the sputtering or backscattering results because the energy loss due to larger impact parameters is negligible as can be seen in Fig. 3a. The situation for the $\mathrm{Si}-\mathrm{Si}$ potential is quite different as shown in Fig. 3a and b. Due to the attractive part of the potential the curves for constant relative energy losses $T / T_{m}$ become different. For a fixed elastic energy loss two curves appear which means that at a fixed energy $E_{0}$ the same energy loss occurs at three different impact parameters $p$. At a fixed energy $E_{0}$ the elastic energy loss decreases at first with increasing impact parameter as for repulsive potentials (see Fig. 7.2 of [4]) and then increases again. The reason for this effect is that the two atoms interact for a larger time which can be seen by looking to the actual trajec- 
tories (see Fig. 2.9b of [4]). These additional energy losses at larger impact parameters decrease the absolute value of the sputtering yield but do not change the dependencies on incident energy and angle of the yields. Because we are interested here mainly in differences of dependencies and not so much in absolute values, we chose also for the Si-Si potential two ring cylinders. Using more ring cylinders increases the computing time. Recoils created at larger impact parameters do not contribute to the sputtering yield because the energy loss is less than the surface binding energy as can be deduced from Fig. 3b. The largest negative scattering angles (laboratory system) are about $-45^{\circ}$ in the laboratory system for all energies down to the surface binding energy [18] so that no orbiting atoms are generated.

Due to the complicated form of the Si-Si potential the calculations need about $30 \%$ more computing time than for the $\mathrm{KrC}$ and the Molière potentials. The use of the ZBL potential affords about $10 \%$ more time than the use of the Molière potential due to its four exponential terms instead of three in Molière and $\mathrm{KrC}$ potentials.

\section{Calculated data and comparison}

All the calculations deal with the bombardment of silicon by silicon. The first example shows the average depth (range) versus the energy of the projectiles for normal incidence in Fig. 4. The dependence is the same for all four potentials applied. The difference is only a few monolayers which is beyond experimental depth resolution. The range increases with a weaker potential; therefore the Molière potential gives the shortest range, the $\mathrm{ZBL}$ potential the largest range at a fixed projctile energy. The data for the Si-Si potential follow the values calculated for ZBL potential at low energies, whereas at higher energies, they are closer to the data calculated with the $\mathrm{KrC}$ potential. This result is expected from the form of the SiSi potential shown in Fig. 1 a.

The dependence of the average depth versus the angle of incidence for $200 \mathrm{eV}$ projectiles is shown in Fig. 5. At

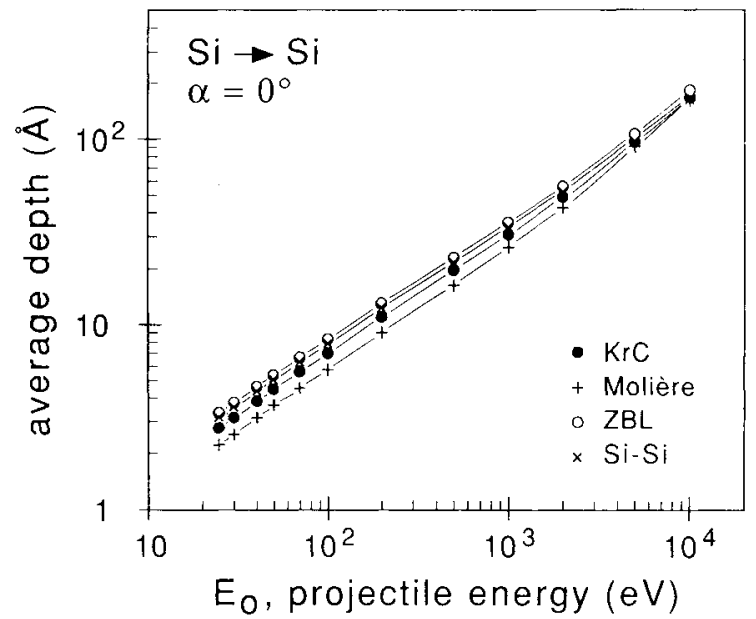

Fig. 4. Average depth of implanted silicon into silicon at normal incidence versus the projectile energy for four interaction potentials

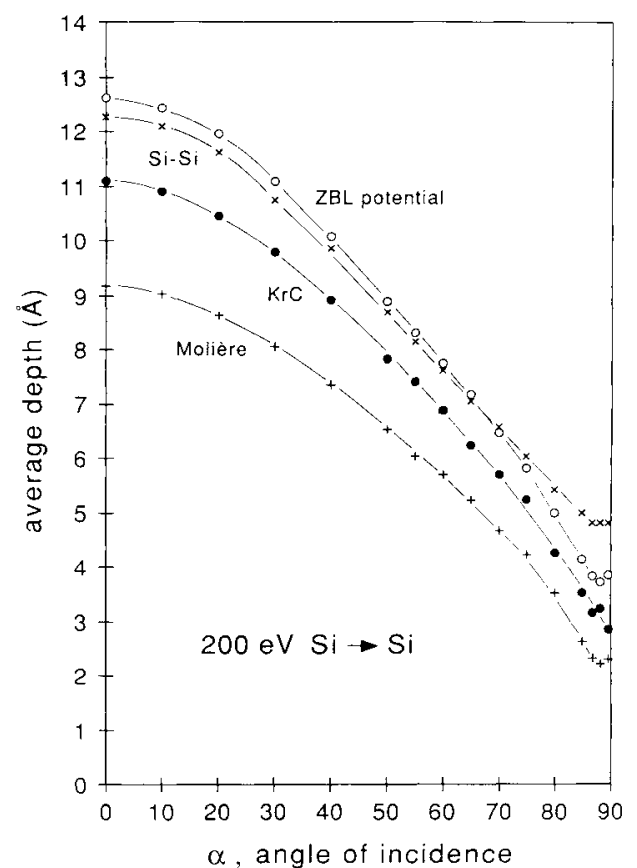

Fig. 5. Average depth of $200 \mathrm{eV}$ silicon implanted into silicon versus the angle of incidence for four interaction potentials

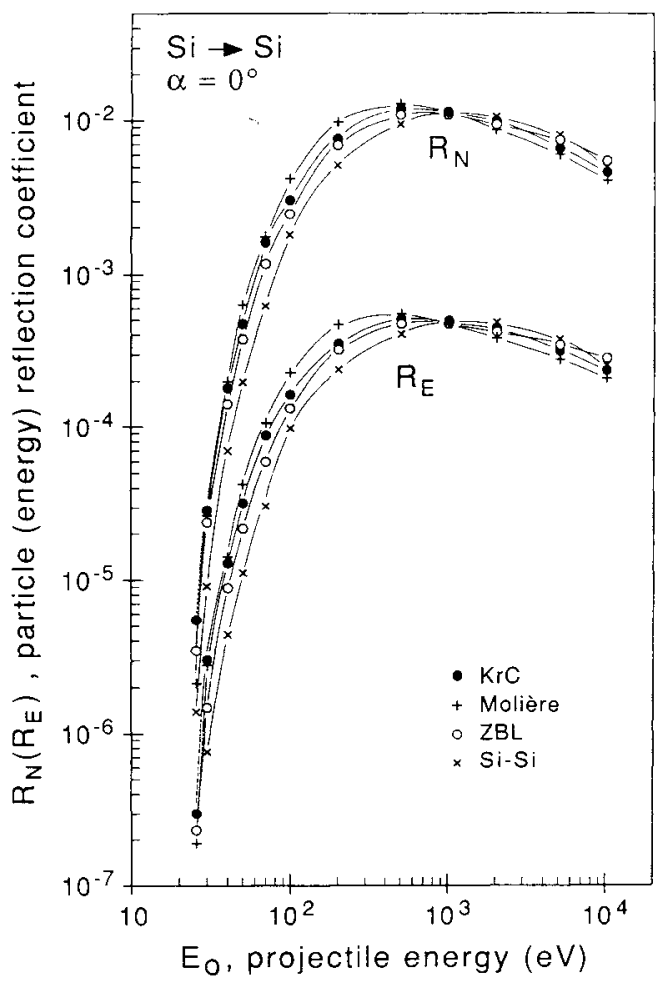

Fig. 6. Particle and energy reflection coefficients, $R_{N}$ and $R_{E}$, respectively, versus the projectile energy. Four different potentials are applied for the bombardment of silicon by silicon at normal incidence, $\alpha=0^{\circ}$

this low energy this dependence does not exhibit a cosine distribution as at high energies. The data for the three repulsive potentials give a similar dependence only shifted in absolute value as discussed above. The data for the Si- 
Si potential show a slightly weaker dependence at large angles of incidence.

The next example is the backscattering of projectils described by the particle and energy reflection coefficients, $R_{N}$ and $R_{E}$, respectively. The general dependence of both reflection coefficients at normal incidence is similar for all four interaction potentials, see Fig. 6. At $1 \mathrm{keV}$ projectile energy the values for $R_{N}$ and $R_{E}$ are the same for all potentials. At lower energies the Si-Si potential exhibits the lowest values for the reflection, whereas at higher energies the situation is reversed. Below $500 \mathrm{eV}$ projectile energy the reflection coefficients for the different potentials can differ by a factor of 2 to 3 , whereas the data for the repulsive potentials alone differ by less than a factor of two (not regarding the small values at $25 \mathrm{eV}$, where the statistical error becomes large).

The angle of incidence dependence of the reflection coefficients is given in Fig. 7 for $200 \mathrm{eV}$ projectiles. The curves calculated with the $\mathrm{Si}-\mathrm{Si}$ potential show systematic lower values, which are about a factor of two lower than for the Molière potential. Whereas all repulsive potentials lead to nearly total particle reflection $\left(R_{N}=1\right)$, the Si-Si potential leads only to $R_{N}=0.75$ near $\alpha=90^{\circ}$. The difference is even larger in the energy reflection.

The last example investigated is the sputtering of target atoms. The sputtering yield verus the projectile energy at normal incidence is shown in Fig. 8. The curves for the repulsive potentials follow each other in absolute values as the potentials which means that the strongest potential gives the highest yields. The curves for the Si-Si

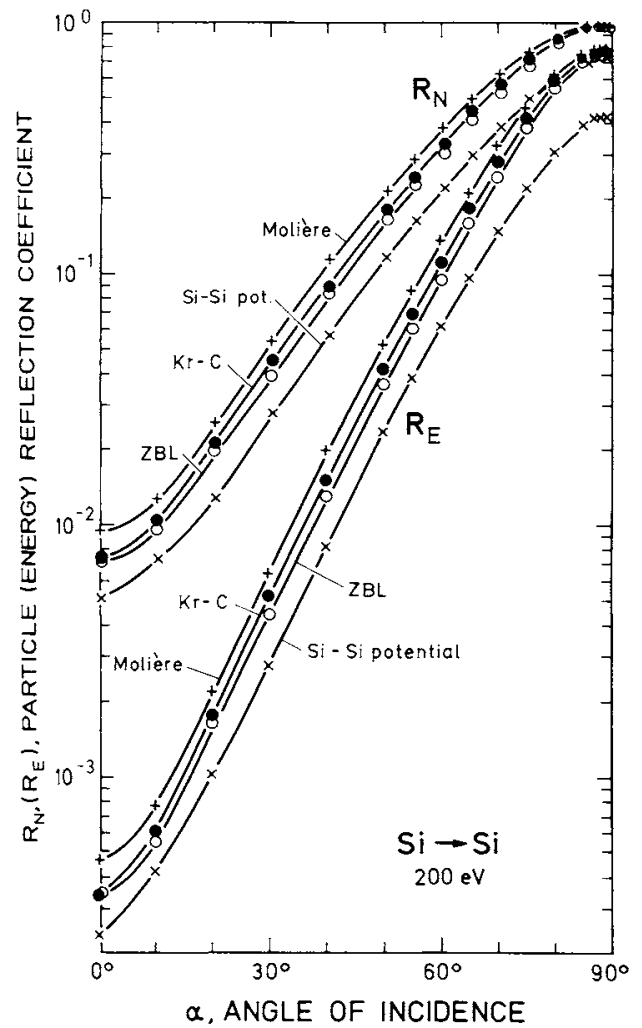

Fig. 7. Particle and energy reflection coefficients, $R_{N}$ and $R_{E}$, respectively, versus the angle of incidence, $\alpha$. Four different potentials are applied for the bombardment of silicon by $200 \mathrm{eV}$ silicon potential is close to the one for ZBL potential at low energies, at higher energies it crosses the curve for the $\mathrm{KrC}$ potential and reaches the same values as for the Molière potential at about $10 \mathrm{keV}$. The difference between the curves for the different potentials is less than

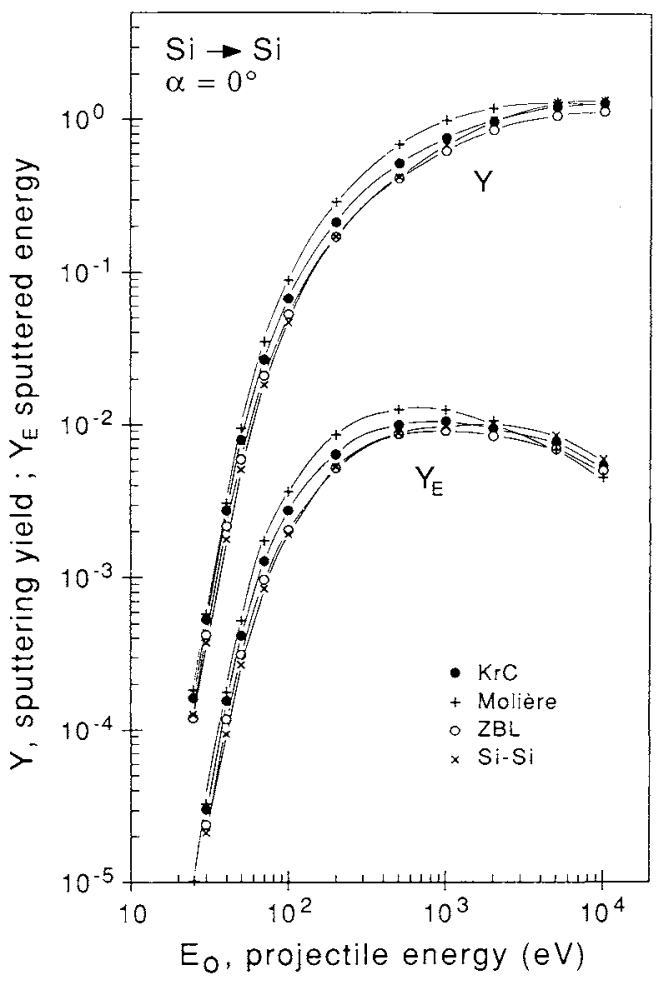

Fig. 8. Sputtering yield, $Y$, versus the projectile energy, $E_{0}$. Four potentials are applied for the bombardment of silicon by silicon at normal incidence, $\alpha=0^{\circ}$

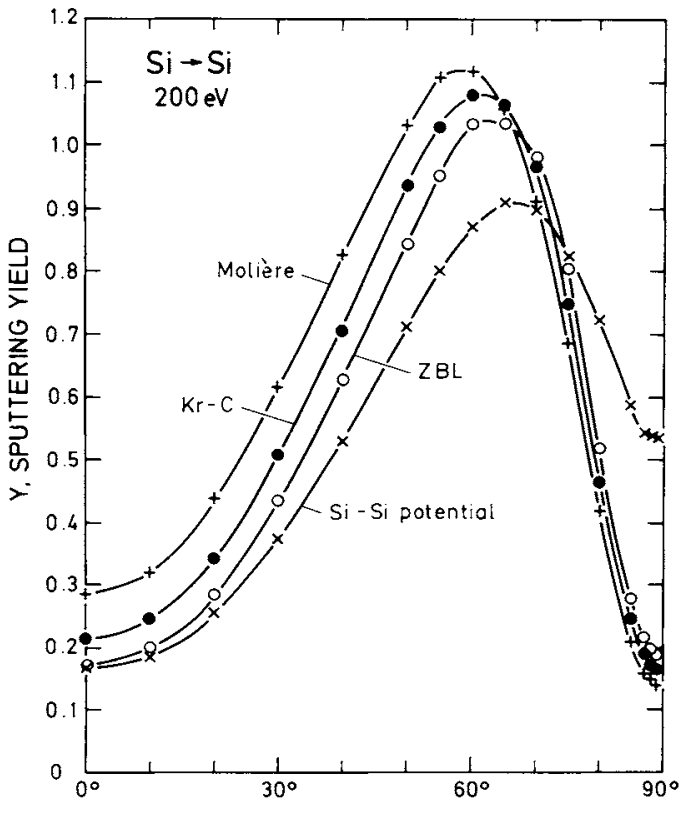

$\alpha$, ANGLE OF INCIDENCE

Fig. 9. Sputtering yield, $Y$, versus the angle of incidence, $\alpha$. Four potentials are applied for the bombardment of silicon by $200 \mathrm{eV}$ silicon 
a factor of two in the whole energy range. A similar result is observed for the sputtered energy, $Y_{E}$.

The angular dependence of the sputtering yield for $200 \mathrm{eV}$ projectiles shown in Fig. 9, exhibits the difference of the data determined by the repulsive potential and the $\mathrm{Si}-\mathrm{Si}$ potential. The main difference is the position of the maximum which is at $\alpha=68^{\circ}$ for the Si-Si potential and around $\alpha=60^{\circ}$ for the repulsive potentials. The biggest difference appears at grazing incidence, where the values for the Si-Si potential are a factor of 3 larger than for the repulsive potentials. This is closely related to the lower reflection coefficients at grazing incidence, see Fig. 7.

\section{Conclusion}

The main result is that a potential with an attractive part shows the same general dependence for the ranges, reflection coefficients and the sputtering yield on the projectile energy and angle of incidence as purely repulsive potentials. There are a few specific deviations for the Si-Si potential compared to the repulsive potentials. The main differences appear at larger angles of incidence as expected. At near grazing incidence the dependence of the range is less steep, the reflection coefficients do not reach values close to unity and the decrease of the sputtering yield is less pronounced (combined with a shift in the position of the maximum).

These differences seem to be large enough to be proven experimentally. But effects of surface roughness are known to be of great importance (Fig. 5 of [19]) so that it will not be easy to check experimentally the effects discussed in this paper. Therefore in most cases it is rea- sonable to apply purely repulsive potentials in simulations of ion-solid interaction.

\section{References}

1. Stillinger, F.H., Weber, T.A.: Phys. Rev. B31, 5262 (1985)

2. Tersoff, J.: Phys. Rev. B37, 6991; 38, 9902 (1988)

3. Harrison, D.E. Jr.: CRC critical reviews in solid state and material sciences, vol. 14, Suppl. 1. Boca Raton: CRC-Press 1988

4. Eckstein, W.: Computer simulation of ion-solid interactions, Springer Series in Materials Science 10. Berlin, Heidelberg, New York: Springer 1991

5. Molière, G.: Z. Naturforsch. A2, 133 (1947)

6. Wilson, W.D., Haggmark, L.G., Biersack, J.P.: Phys. Rev. B15, 2458 (1977)

7. Ziegler, J.F., Biersack, J.P., Littmark, U.: The stopping and range of ions in solids, vol. 1. New York: Pergamon Press 1985

8. O'Connor, D.J., Biersack, J.P.: Nucl. Instrum. Methods B15, 14 (1986)

9. Heinemann, D., Rosén, A., Fricke, B.: Phys. Scr. 41, 692 (1990)

10. Hackel, S., Fricke, B., Heinemann, D., Kolb, D., Yang, L.: GSI-90-1, p. 165 (1990)

11. Biersack, J.P., Eckstein, W.: Appl. Phys. A34, 73 (1984)

12. Oen, O.S., Robinson, M.T.: Nucl. Instrum. Methods 132, 647 (1976)

13. Lindhard, J., Scharff, M.: Phys. Rev. 124, 128 (1961)

14. Biersack, J.P., Haggmark, L.: Nucl. Instrum. Methods 174, 257 (1980)

15. Davis, P.J., Polansky, I.: In: Handbook of mathematical functions, chap. 25, p. 889. Abramowitz, M., Stegun, I.A. (eds.). New York: Dover 1965

16. Robinson, M.T., Torrens, I.M.: Phys. Rev. B9, 5008 (1974)

17. Robinson, M.T.: Tables of Classical Scattering Integrals, U.S. Atomic Energy Emission, Report ORNL-4556, 1970

18. Schlager, H.G., Eckstein, W.: IPP-Report 9/69, Garching, 1991

19. Eckstein, W., Bohdansky, J., Roth, J.: Nuclear fusion supplement, vol. 1, IAEA, Vienna, 1991 